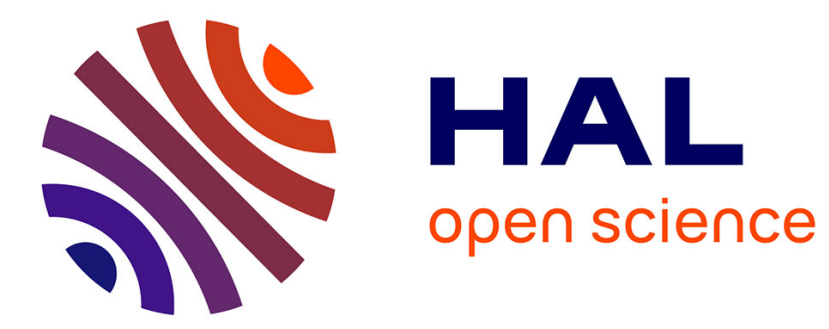

\title{
THE DIFFUSION OF FISSION GAS ATOMS AND BUBBLES THROUGH URANIUM DIOXIDE
}

\author{
C. Catlow, M. Wood
}

\section{To cite this version:}

C. Catlow, M. Wood. THE DIFFUSION OF FISSION GAS ATOMS AND BUBBLES THROUGH URANIUM DIOXIDE. Journal de Physique Colloques, 1976, 37 (C7), pp.C7-428-C7437. 10.1051/jphyscol:1976797 . jpa-00216957

\section{HAL Id: jpa-00216957 https://hal.science/jpa-00216957}

Submitted on 1 Jan 1976

HAL is a multi-disciplinary open access archive for the deposit and dissemination of scientific research documents, whether they are published or not. The documents may come from teaching and research institutions in France or abroad, or from public or private research centers.
L'archive ouverte pluridisciplinaire HAL, est destinée au dépôt et à la diffusion de documents scientifiques de niveau recherche, publiés ou non, émanant des établissements d'enseignement et de recherche français ou étrangers, des laboratoires publics ou privés. 


\title{
THE DIFFUSION OF FISSION GAS ATOMS AND BUBBLES THROUGH URANIUM DIOXIDE
}

\author{
C. R. A. CATLOW $\left(^{*}\right)$ and M. H. WOOD \\ Theoretical Physics Division, AERE, Harwell, Oxfordshire OX11 ORA, U. K.
}

\begin{abstract}
Résumé. - Nous présentons d'abord une étude des mécanismes de capture et de migration des atomes de fission à l'état gazeux : $\mathrm{Kr}$ et $\mathrm{Xe}$ dans $\mathrm{UO}_{2}$. Notre approche consiste à combiner les résultats des calculs énergétiques des procédés considérés, avec les informations provenant des études expérimentales sur la diffusion des gaz, principalement les données de Felix et Mickeley. Nos modèles sont notablement différents de ceux précédemment présentés pour la diffusion des gaz rares dans les cristaux ioniques. Nos propositions sont les suivantes : la capture a lieu dans les agrégats de lacunes ; la diffusion de Xe est principalement due à des mécanismes qui ne sont pas liés aux défauts du réseau; nous montrons également que la libération des atomes gazeux dans les sites interstitiels n'est pas importante. Différents mécanismes sont suggérés pour la capture et la diffusion des deux atomes : ceci provient de leur différence de taille. Nos résultats rationalisent les principales observations de l'étude expérimentale de Felix et Mickeley et soulignent l'importance de la composition chimique du combustible nucléaire pour les coefficients de diffusion des gaz. Les contradictions entre ce travail et les études précédentes résumées par Matzke, pourraient provenir, à notre point de vue, d'un degré plus important de détérioration par radiation des cristaux utilisés dans les travaux antérieurs.

Nos propositions pour les mécanismes de diffusion d'un gaz monoatomique sont ensuite utilisées pour une discussion de la migration de bulles gazeuses. A ce stade nous essayons de réconcilier les contradictions apparentes qui existent, entre différentes études expérimentales, certaines faisant état d'un mécanisme de diffusion en volume tandis que d'autres sont compatibles avec un mécanisme de diffusion en surface. Nous suggérons que pour certains cas le mécanisme de diffusion en surface peut être supprimé à cause de l'accumulation sur la surface de la bulle d'impuretés qui diffusent avec les atomes gazeux vers les noyaux de la bulle pendant la naissance de celle-ci.
\end{abstract}

\begin{abstract}
We report first a study of the trapping and migration mechanisms of the fission gas atoms, $\mathrm{Kr}$ and $\mathrm{Xe}$ in $\mathrm{UO}_{2}$. Our approach is to combine the results of calculations of the energetics of the processes we consider, with the evidence from experimental studies of gas release - principally the data Felix and Miekeley. Our models differ markedly from those previously presented in discussions of rare gas diffusion in ionic crystals. We propose that trapping occurs at vacancy aggregates, and that Xe diffusion occurs predominantly by mechanisms which are not assisted by lattice defects; also detrapping of gas atoms into interstitial sites is shown to be unimportant. Different mechanisms are suggested for the trapping and diffusion of the two atoms; this difference arises from their different sizes. Our results rationalize the principal observations of the experimental study of Felix and Miekeley and emphasize the importance of the chemical composition of the fuel on gas diffusion coefficients. The contradictions between this work and the earlier studies summarized by Matzke is, we suggest, possibly due to the higher levels of radiation damage which may have been present in the crystals used in the latter work. Our proposals for the mechanisms of diffusion of single gas atoms are then used in a discussion of gas bubble migration. Here we attempt to reconcile apparent contradictions between different experimental studies, some of which indicate volume diffusion while others are compatible with a surface diffusion mechanism. We suggest that in some cases surface diffusion may be suppressed owing to the accumulation on the surface of the bubble of impurities which diffuse with the gas atoms to the bubble nuclei during bubble growth.
\end{abstract}

1. Introduction. - Owing to the technological importance of the behaviour of fission gases in $\mathrm{UO}_{2}$ a large number of experimental studies have been reported in this field. These have concerned both the diffusion of gas atoms $\mathrm{Kr}$ and $\mathrm{Xe}[1,2]$ and the nucleation and migration of fission gas bubbles [3-9]. In neither area however, have gas migration mechanisms

(*) Present address : Dept. of Chemistry, University College London, Gower St., London WCl E6BT, been clearly identified. Thus in the present paper we examine these problems using theoretical methods for calculating defect energetics which have been successfully employed in other studies of the transport properties of solids $[10,11,12]$, including $\mathrm{UO}_{2}[13,14]$. We discuss both the trapping and migration of the fission gases, and the transport mechanism of bubbles ; and we pay particular attention to the effects of stoichiometry and impurities. The models we present differ very considerably from those previously used in discussing 
rare gas diffusion in ionic crystals [15]. We propose that trapping of single gas atoms occurs at vacancy aggregates rather than at single vacancy sites ; and migration occurs by non-defect assisted mechanisms which do not involve de-trapping to interstitial sites. Our models lead to a coherent account of the experimental data of Felix and Miekeley [1] ; although problems remain in rationalising the data reviewed by Matzke [2]. Our results on the diffusion of single gas atoms are also of value in our subsequent discussion of bubble migration mechanisms.

The essential details of our calculations are summarized in the following two sections, after which we present the analysis of our results and our detailed mechanistic proposals. We then conclude with brief suggestions as to further experimental work.

2. Computational Methods and Lattice Potentials. We present no details here as the methods used in our calculations (which all employed the Hades program) have been discussed elsewhere in this conference [10]. Several studies $[12,13,14]$, including the others in this conference $[10,11]$, have shown the reliability of these methods in studies of defect energetics; indeed the limitations on the accuracy of the calculated defect energies are entirely attributable to uncertainties in the potentials used in describing the interaction of lattice ions and of lattice and defect species.

Our lattice potential for $\mathrm{UO}_{2}$ is taken from the study of Catlow [14] where a fully ionic model is assumed for the crystal. We describe short range interionic potentials using Born-Mayer or Buckingham potentials; and the lattice potential uses a shell model [20] treatment of ionic polarisation, whose advantages for defect studies are discussed elsewhere [10].

We also require potential parameters for the interaction of the two gases $\mathrm{Kr}$ and Xe with both uranium and oxide anions. Here we used the approximate theoretical techniques developed by Wedepohl [16] and Gordon and Kim [17]. These methods have been extensively used in studies of rare gas interactions [17] and of ionion potentials in the alkali halide crystals [18] ; in both cases good agreement is found between calculated and experimental interaction energies. To obtain our gaslattice potential parameters, we fitted our calculated interaction energies to a Born Mayer potential ; the parameters are presented and discussed in greater detail elsewhere [19]. The method, although approximate, should be superior to methods previously used in parameterising gas lattice potentials [15]; these were based on less accurate theoretical techniques and on approximate estimates of gas atom radii.

Finally, we require shell model parameters to describe the polarisation properties of the gas atom. Here we used the results of Dick and Overhauser [20].

3. Results. - 3.1 TraP Sites. - In table I we present our calculated energies for the two gases at a variety of sites in the fluorite lattice of $\mathrm{UO}_{2}$. The ener-

TABLE I

$\mathrm{Kr}$ and $\mathrm{Xe}$ in $\mathrm{UO}_{2}$ defect energies (i. e. energy to introduce the gas atom from infinity, and in the case of the trapped atoms, to create the trapping site by displacement of lattice ions to infinity)

Site

Interstitial

Anion vacancy

Cation vacancy

Neutral trivacancy (Fig. 1)

Charged trivacancy (Fig. 2)

Tetravacancy (Fig. 3)

(*) Estimated, see text.

gies given for gas atoms trapped at vacancy sites include the formation energy of the trap, that is the energy to remove the appropriate lattice ions to infinity. We examined trapping at vacancy aggregates in addition to isolated vacancy sites. Three such aggregates were considered : the neutral trivacancy (comprising one cation and two anion vacancies) shown in figure 1 ; the

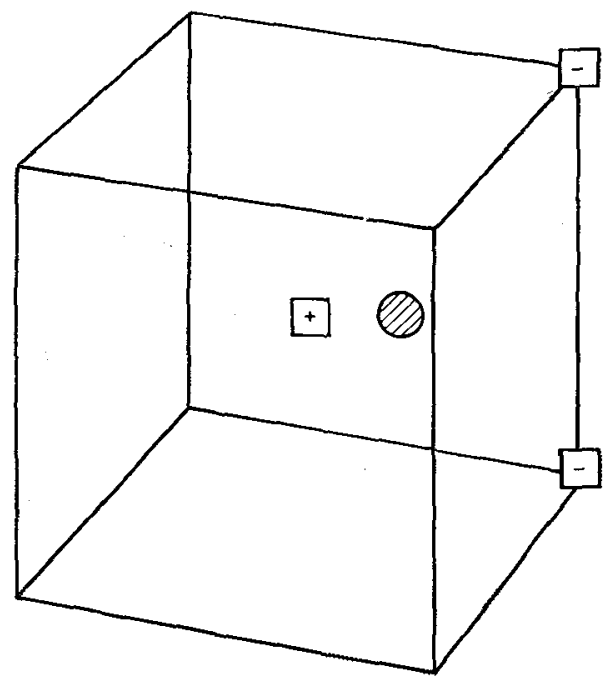

FIG. 1. - Neutral trivancy and gas atom. ĐCation vacancy. $\Theta$ Anion vacancy. Gas atom (Corners of cube indicate regular anion sites in fluorite lattice).

charged trivacancy (one anion plus two cation vacancies) shown in figure 2 ; and the tetravacancy (two cation plus two anion vacancies) - see figure 3 . In one case, that of $\mathrm{Xe}$ in the charged trivacancy, the calculation failed to converge, because an excessive dipole moment developed on the highly polarisable Xe atom. We therefore estimated the result here by assuming that the trapping energy (i. e. the energy to remove the gas 


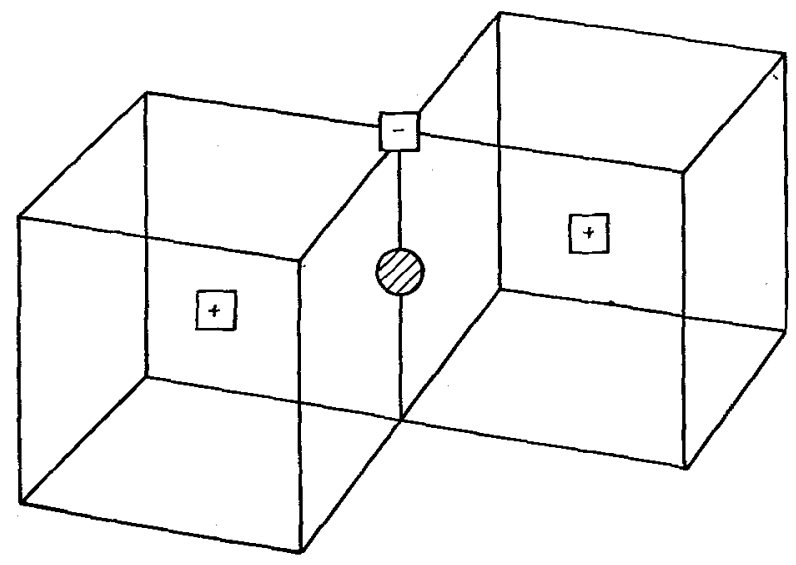

Fig. 2. - Charged trivacancy and gas atom. $⿴$ Cation vacancy. $G$ Anion vacancy. Gas atom(Corners of cube indicate regular anion sites in fluorite lattice).

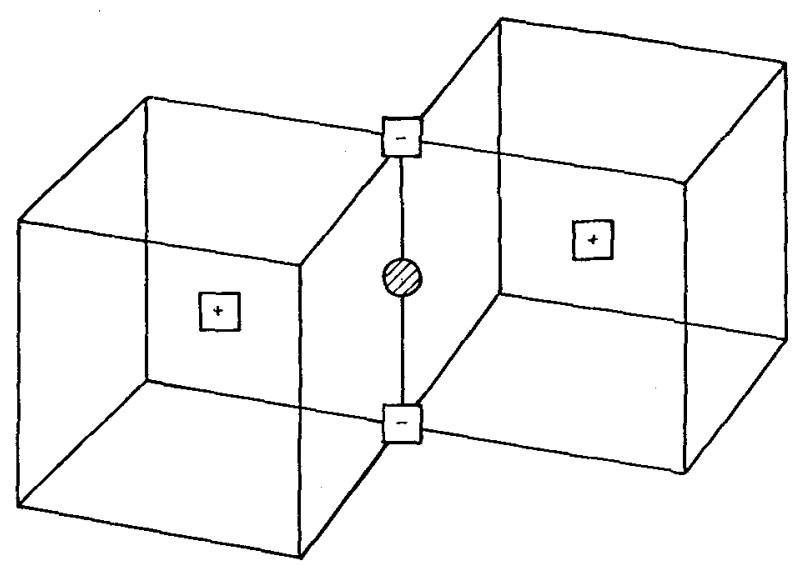

FIG. 3. - Tetravacancy. $⿴$ Cation vacancy. $\square$ Anion vacancy. Gas atom (Corners of cube indicate regular anion sites in fluorite lattice).

atom to a pre-existing trapping site) for $\mathrm{Xe}$ was the same for the charged trivacancy as for the tetravacancy (which differs only by the presence of an extra anion vacancy). The errors introduced by this approximation are discussed in the following section.

We also performed calculations on isolated trap sites which we report in our more detailed account [19]. These values together with results obtained previously for basic defect energies (Schottky and Frenkel formation) in $\mathrm{UO}_{2}$, allow us to deduce the trap Arrhenius energies, $E_{\mathrm{A}}$, which govern trap concentrations, according to the expression :

$$
x=A \exp \left(-E_{\mathrm{A}} / k T\right)
$$

where $A$ is a constant. These energies are dependent on the stoichiometry of the oxides. Further details of their derivation are given elsewhere [19], but values are not reported here as the energies are only an intermediate stage in the derivation of the more significant gas formation energies, which are discussed below and reported in table II.

From the results in table $I$ and from the energies of formation of isolated trap sites we may also deduce the trapping energies, $E_{\mathrm{T}}$, defined above (see ref. [19]). Finally we obtain the gas formation energy, $E_{G}$, defined as the trap Arrhenius energy, $E_{\mathrm{A}}$ minus the trapping energy, $E_{\mathrm{T}}$. Calculated values of $E_{\mathrm{G}}$ are given in table II for the two gases, and for different stoichiometries. We may use these energies to deduce the trapping site, for crystals in thermal equilibrium: a negative value indicates that trapping is favourable, as the energy released on trapping the gas atom from the interstitial site outweighs the energy required to form the trap; conversely, a positive value shows that trapping will not occur.

3.2 Gas Migration. - We first examined interstitial migration mechanisms. The activation energies we calculated for both gas atoms were prohibitively high - at least $5 \mathrm{eV}$. And on comparing these results with those presented below, one may conclude that detrap. ping of gas atom to interstitial sites will not play an important role in the diffusion of either gas atom.

\section{TABLE II}

Gas formation energies, $E_{\mathrm{G}}$ (i. e. trap Arrhenius energy $\left(E_{\mathrm{A}}\right)$ - trapping Energy $\left.\left(E_{\mathrm{T}}\right)\right)$

$$
\text { Trap }
$$

Anion vacancy
Cation vacancy
Neutral trivacancy
Charged trivacancy

Tetravacancy
Anion deficient $\mathrm{UO}_{2}$

$\overline{\mathrm{Kr}} \quad \overline{\mathrm{Xe}}$

Formation energy (eV)

Stoichiometric

$\overline{\mathrm{Kr}} \quad \overline{\mathrm{Xe}}$

0.8

0.5

$-1.7$

$-3.3$

0.17

$-3.43$

8.66

9.95

3.84
6.31
1.01

$-1.46$
Anion excess $\mathrm{UO}_{2}$

$\overline{\mathrm{Kr}} \quad \overline{\mathrm{Xe}}$

3.3

$-6.7-8.6$

0.17

$-3.43$

$-5.45$

$-6.34$

$-3.99-6.46$ 
Three other mechanisms were then considered; we report results in table III. The trivacancy mechanism is illustrated in figure 4. It is a many-step process comprising first an interchange of the gas atom and a neighbouring lattice cation via interstitial sites as shown in figure 4 . The subsequent steps in the diffusion process consist of the very rapid migration of the anion vacancies characteristic of the fluorite lattice, around cation vacancy/gas complex. Together, these processes effect migration of the gas atom-vacancy complex. The first step is probably rate determining, as we calculate an activation energy of $\sim 5 \mathrm{eV}$ as reported in table III ; while the experimental value for the activation energy for anion vacancy jumps (see Catlow and Lidiard [13]), which should be close to that for the second step in our mechanism, is $\sim 0.25 \mathrm{eV}$.

\section{TABLE III}

\section{Activation energies}

Mechanism

-

Neutral trivacancy $\left({ }^{a}\right)$

Charged trivacancy and tetravacancy $(b)$

\section{Energies (eV)

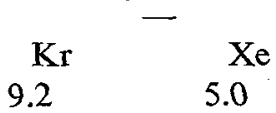

$2.5(*)$

$2.5(*)$

(a) Proposed saddle point shown in figure 4.

(b) Proposed saddle point shown in figure 5.

$\left(^{*}\right)$ This value was obtained from experimental data of Matthews (see text). It is the Arrhenius energy for lattice ion diffusion in hyperstoichiometric $\mathrm{UO}_{2+x}$, which will be very close to the activation energy for a cation vacancy jump (see Catlow [14] ; Matthews [21]).

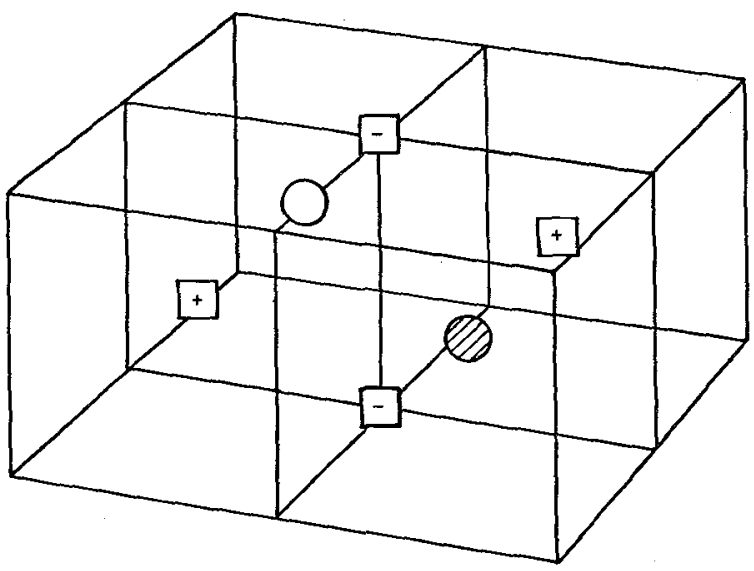

FIG. 4. - Saddle point for neutral trivacancy diffusion mechanism. $⿴ 囗 十$ Cation vacancy. $日$ Anion vacancy. OMigrating cation passing through interstitial site. Gas atom passing through interstitial site.

Related to the above are the charged trivacancy and tetravacancy mechanisms. Here the gas atom moves between the two cation vacancies of the trap (a process which is either unactivated or has a very low activation energy) and one of the vacancies jumps round the second vacancy occupied by the gas atom, via the saddle point shown in figure 5. Readjustment of the anion vacancies around the cation vacancy complex will again be rapid. We were, however, unable to perform a calculation on this rate determining cation vacancy jump, as the low symmetry of the saddle point precludes a reliable calculation. Furthermore when calculations were attempted they failed due to a divergence in the polarisation energy of the gas atom. However, as mentioned above, a reasonable estimate of the activation energy for this process may be obtained from the experimental value for isolated vacancy jumps, which we therefore report in table III. The value may well be an overestimate, as the polarisable gas atom will stabilise the low symmetry saddle point.

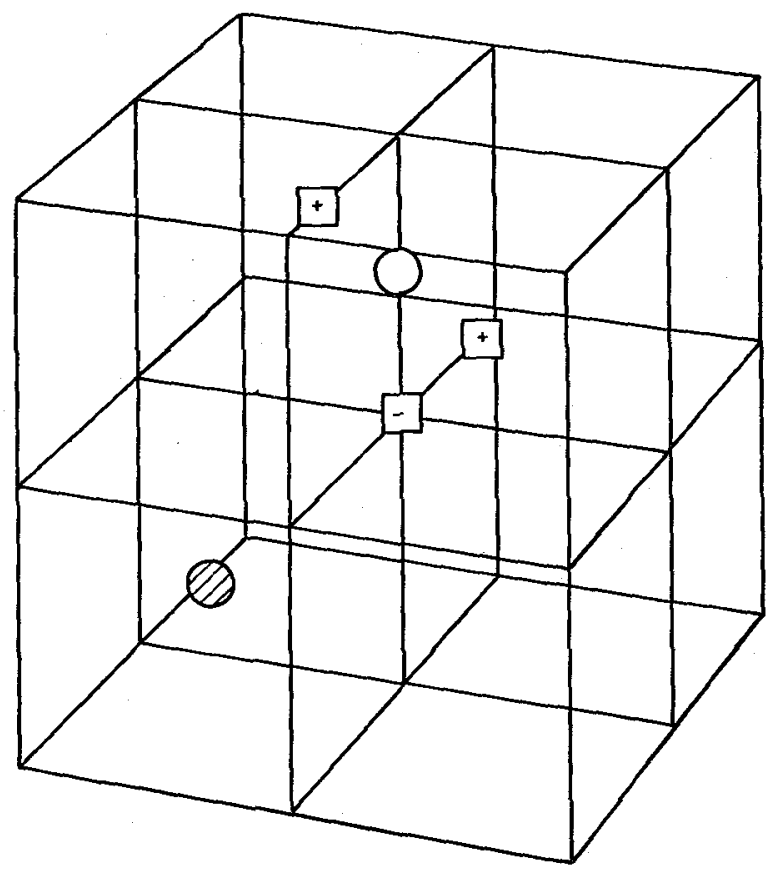

FIG. 5. - Proposed saddle point for charged trivacancy diffusion mechanism (Same saddle point for tetravacancy mechanism, excepting the presence of an additional vacancy at site A). O Migrating cation. $⿴ 囗 十$ Cation vacancy. $\boxminus$ Anion vacancy. Gas atom at vacant cation site

4. Discussion. - 4.1 GAS ATOM TRAPPING AND DIFFUSION. - As remarked in section (3.1), we may deduce trap sites from the gas formation energies given in table II. The resulting predictions are summarized in table IV, where we also include our proposed migration mechanisms for the gases in the different regions of stoichiometry. To estimate these we used both calculated activation energies, and experimental Arrhenius energies for lattice ion diffusion where appropriate. The mechanism with the lowest Arrhenius energy is then taken to predominate. We now consider these predictions in detail for two gas atoms separately.

4.1.1 Xenon diffusion. - Our results suggest that in hypostoichiometric (anion deficient) and stoichiometric $\mathrm{UO}_{2}, \mathrm{Xe}$ will be trapped at neutral trivacancies. For 


\section{TABLE IV}

Trapping sites, Arrhenius energies and migration mechanisms

$\frac{\text { Gas }}{\mathrm{Kr}}$ Mechanism

Energy $(\mathrm{eV})$

Xe Mechanism

Energy $(\dagger)$

(eV)
5.0
$(6.0)$
Stoichiometric $\mathrm{UO}_{2}$

Cation vacancy trapping. Diffusion controlled by lattice cation migration.

$$
4.0\left(^{*}\right)
$$

Neutral trivancy trapping. Diffusion controlled by lattice cation migration
Anion excess $\mathrm{UO}_{2}$

Cation vacancy trapping. Diffusion controlled by lattice cation migration.

\section{$2.5\left({ }^{*}\right)$}

Trapping at either cation vacancies or charged trivacancies ; experimental data favours the latter. Diffusion by nondefect assisted mechanism via saddle point shown in figure 5 .

( $\dagger$ ) Activation energy for anion vacancy diffusion (see Catlow et al. [23]).

(*) Experimental Arrhenius energies for lattice cation diffusion (see Matthews [21]).

$(\dagger)$ Experimental Arrhenius energies for Xe diffusion [1] given in brackets.

$4.0(*)$ the hyperstoichiometric (anion excess) crystal, however, a single cation vacancy trap is predicted. However, we recall that the energy of a gas atom trapped in charged trivacancy was based on the assumption that the trapping energy at this site was the same as for the tetravacancy. Although this approximation was necessary in the absence of any alternative, we believe that it may lead to significant errors, as the polarisation energy (which is zero for the tetravacancy trap) may be arge in the charged trivacancy; for the atom in the neutral trivacancy a value of $\sim 1.7 \mathrm{eV}$ was calculated for this energy. In view of this uncertainty we cannot discount the charged trivacancy trap for the hyperstoichiometric crystals.

The most extensive and detailed experimental data with which to compare our results were obtained by Felix and Miekeley [1] who studied gas release from Xe implanted crystals of $\mathrm{UO}_{2}$. The two most important features of their results were as follows :

(i) Variation of the Xe diffusion coefficient with stoichiometry. - A strong dependence was observed; the Arrhenius energy of the diffusion coefficient, $D$, decreases by $\sim 4 \mathrm{eV}$ on passing from hypo to hyperstoichiometric crystals. These energies are given in table IV, and we note satisfactory agreement with our predicted values.

However, although diffusion is dependent on the composition of the host lattice in the sense discussed above, within each region of stoichiometry the diffusion coefficient is independent of composition; that is although diffusion is markedly altered on passing from hyper to hypostoichiometric $\mathrm{UO}_{2}$, within either of these two regions of composition $D$ does not vary. This important observation is in line with our predicted mechanism for hypostoichiometric $\mathrm{UO}_{2}$. Here we have suggested the non-defect assisted trivacancy mechanism ; and only for such a non-defect assisted process can we expect a diffusion coefficient that is independent of composition. The same criterion suggests that in the hyperstoichiometric crystal, the charged trivacancy will predominate. In this case the Xe atom may also diffuse by a non-defect assisted mechanism ; in contrast, the alternative proposal of cation vacancy trapping would require a vacancy assisted diffusion mechanism which would lead to a value of $D$ dependent on the composition of the crystal.

(ii) Time Dependence of Diffusion Coefficients. Felix and Miekeley [1] in their diffusion anneals observed an initial period of time dependent diffusion coefficients; preliminary oxidation anneals showed that this could not be attributed to time dependence of the composition of the $\mathrm{UO}_{2}$. Our proposals immediately account for this observation. All the traps we have suggested contain cation vacancies; but cation diffusion coefficients are low in $\mathrm{UO}_{2}$ (see Matthews [21]), hence the rate of trap formation will be low. The initial time dependence of the diffusion coefficient is therefore a consequence of the slow rate of gas atom trapping. We should note that simpler models of trapping in anion vacancy sites, although successful in discussing Ar diffusion in $\mathrm{CaF}_{2}$ [22], are not acceptable for $\mathrm{Xe}$ diffusion in $\mathrm{UO}_{2}$; anion diffusion is rapid in $\mathrm{UO}_{2}[13$, 
$14,21]$ and hence anion vacancy trapping would not lead to an initial period with a time dependent diffusion coefficient due to slow gas atom trapping.

Felix and Miekeley also examined the effect of sudden changes in the equilibrium oxygen pressure during the diffusion anneals. The most detailed results were obtained for hyperstoichiometric $\mathrm{UO}_{2+x}$ for which the equilibrium oxygen pressure was suddenly reduced to a value appropriate for the stoichiometric oxide. At higher temperatures $\left(\sim 1250^{\circ} \mathrm{C}\right)$ a slow gradual reduction of the diffusion coefficient was observed, from the value characteristic of $\mathrm{UO}_{2+x}$ to one appropriate for stoichiometric $\mathrm{UO}_{2}$. At lower temperatures $\left(950^{\circ} \mathrm{C}\right)$, an immediate response occurs to a release rate intermediate between those for the two oxides. The rate then remains constant, but is further reduced to the value for stoichiometric $\mathrm{UO}_{2}$ on raising the temperature to $1250^{\circ} \mathrm{C}$.

To explain these results, our models must include two features. First, the trap in hyperstoichiometric $\mathrm{UO}_{2+x}$ must contain a different number of cation vacancies from that in the trap present in the stoichiometric crystal; only then can we rationalise the slow change in the diffusion coefficient, after changing the oxygen partial pressure, to the value appropriate to the stoichiometric crystal. Second, the immediate response observed at low temperatures to the change in oxygen partial pressure indicates a change, on removing excess oxygen, in the number of anion vacancies present in the trap ; the response is immediate owing to the high mobility of anionic defects.

The existence of the charged trivacancy containing two cation vacancies as the predominant trap in the hyperstoichiometric crystal accords with both these points. For in the stoichiometric crystal we predict a trap (the neutral trivacancy) in which only one cation vacancy (in addition to two anion vacancies), is present. The slow change of the diffusion coefficient to the value for stoichiometric $\mathrm{UO}_{2}$ after reduction of oxygen partial pressure follows therefore from the different number of cation vacancies present in the trap. The immediate response, however, is, we suggest, due to the capture by the charged trivacancy of an anion vacancy on removing the excess oxygen, to give the tetravacancy trap shown in figure 3 . The gas formation energies - see table II - suggest that this will occur. At low temperatures cation diffusion is too slow to allow conversion of this defect to the simpler trivacancy ; but on raising the temperature, the conversion slowly occurs.

As remarked in section (3.2), we were unable to perform detailed calculations on the activation energy for the traps containing two cation vacancies. However, it seems plausible that small differences would exist between the activation energies for migration of the charged trivacancy and tetravacancy traps in accordance with the observed change at low temperatures in the diffusion coefficient on passing from hypostoichiometric to stoichiometric crystals.
Our proposals thus explain the main features of the results of Felix and Miekeley [1]. However, the conclusions of the latter study were in marked contrast to those of earlier work reviewed by Matzke [2] in which little effect of release rate upon composition was observed. It is clearly not possible for us to explain these contradictions between the results of different experiments. But we suggest that the origin of the discrepancies may be in the different levels of radiation damage present in the crystals. In the experiments of Felix and Miekeley [1] such damage should be unimportant, owing to the long diffusion anneals used in their experiments. It is, however, possible that in the work reviewed by Matzke [2], radiation damage may not have fully annealed out. The consequent existence of an extrinsic trap distribution will profoundly modify the release rates of fission gases. In particular the effects of stoichiometry may be far less pronounced, as these operate via the perturbation of defect equilibria by the defects created on deviating from the stoichiometric composition. The failure in some studies (discussed by Matzke) to observe significant effects of composition on release rates, may be due to the presence in the materials studied of an appreciable concentration of traps created by radiation damage; and this factor should clearly be borne in mind when analysing the experimental data.

A final point concerns the effect of impurities on $\mathrm{Xe}$ diffusion. Aliovalent ions (mainly low valent) are normally present in $\mathrm{UO}_{2}$. Furthermore, their concentration increases during the burn up of $\mathrm{UO}_{2}$ fuels in the reactor, as aliovalent ions (again largely of low valence) are created as fission products. We believe that low valent ions may have an important effect on gas diffusion coefficients in the hypostoichiometric crystals. These ions will bind anion vacancies, as their effective charge is opposite to that of the latter defects. Furthermore the impurity-vacancy complex will bind to the trivacancy-gas complex, as the presence of additional vacancies will lower the strain energy of gas atom. The type of structure envisaged is shown in figure 6 .

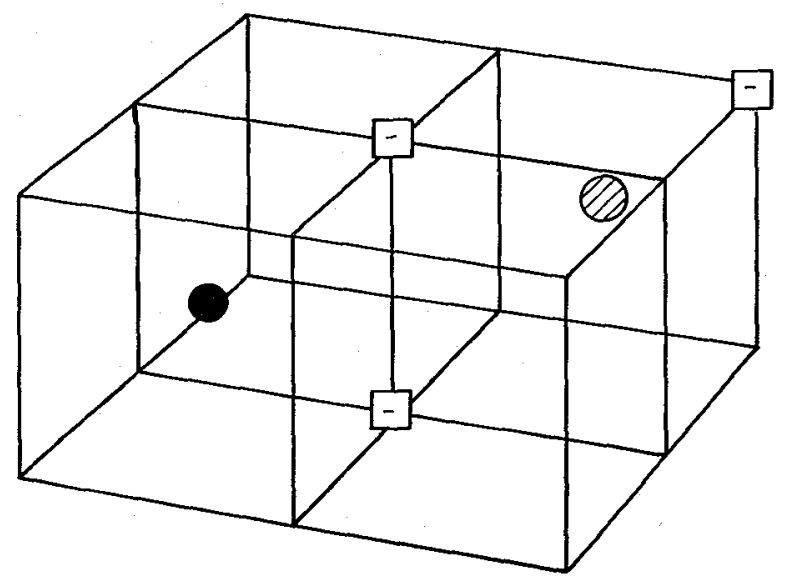

FiG. 6. - Complex of impurity, gas atom and anion vacancies. Impurity atom at vacant cation site. Gas atom at vacant cation site. $\boxminus$ Anion vacancy. 
Detailed calculations on this structure were, however, not possible owing to its low symmetry.

Such structures are important as they may allow more rapid diffusion of gas atoms. The diffusion mechanism proposed for the gas - trivacancy complex was shown in figure 4 . It involves the gas atom passing through an interstitial site. The strain energy here will be greater than when the atom occupies a cation vacancy site. Thus the presence of the additional vacancies, bound by the impurities, should result in a greater reduction of the energy of saddle points of the type shown in figure 4 than in the energies of the ground state illustrated in figure 6 . We confirmed, in separate calculations, that the presence of additional anion vacancies results in a greater reduction in strain energies in an interstitial site compared with a cation vacancy. We performed simple calculations in which we examined the strain energy of a gas atom in both cation vacancy and interstitial site, both for isolated sites, and for those sites surrounded by anion vacancies. The results suggested that the contribution of strain energy to the activation energy for gas diffusion via interstitial sites is reduced by anion vacancies by $\sim 0.3 \mathrm{eV}$ per vacancy.

It is, however, not sufficient to show that gas atoms may diffuse more rapfidly around impurities. Equally rapid migration of the impurity about the gas atom is necessary if the latter is not to be rate determing. We examined a mechanism in which the gas atom and impurity exchange positions. Our calculations, however, again failed to converge due to the polarisation energy of the Xe atom. But if there is a large favourable contribution to the saddle point energy from polarisation, then this suggests that the activation energy may be low. We propose therefore that gas atoms and impurities may move together through the crystal, with a lower activation energy than for the isolated complex of the trivacancy and gas atom.

The mechanisms we have discussed will not operate in stoichiometric or hyperstoichiometric crystals, as excess vacancies are not present. But in hypostoichiometric oxides, our results suggest that low valence impurities may enhance gas diffusion and that the diffusing gas atom carries the impurity with it through the crystal. We will show in section (4.2) that if this impurity-gas mechanism does indeed dominate in the hypostoichiometric oxides, then important effects may follow regarding the diffusion of the bubbles nucleating via diffusion of single gas atoms.

4.1.2 Krypton release. - The smaller size of the $\mathrm{Kr}$ atom leads to predicted trap sites which differ from those discussed above for Xe. In stoichiometric and hyperstoichiometric oxides, the cation vacancy site is predicted ; for the latter oxides, the charged trivacancy (for which a calculation is now possible) is now unimportant, owing to the much lower polarisation energy of $\mathrm{Kr}$ compared with Xe. Cation vacancy trapping will require defect assisted diffusion which should be distinguishable from the non-defect assisted mechanism discussed above for Xe.

The most radical difference between predictions for two gases are, however, for the hypostoichiometric material; trapping into anion vacancies is favoured for $\mathrm{Kr}$. Due to the smaller size of the $\mathrm{Kr}$ atom, the extra reduction in strain energy on trapping into a trivacancy compared with an anion vacancy is not sufficient to outweigh the formation energy of the former ; no formation energy, we recall, is required for the anion vacancy trap in the reduced oxide.

This difference in the trap site for $\mathrm{Kr}$ in $\mathrm{UO}_{2-x}$ will result in major changes in the behaviour of gas release compared with that observed for Xe. Diffusion could occur by detrapping of gas atoms into interstitial sites followed by interstitial gas diffusion. However, our results suggested a high value for the activation energy for gas interstitial migration. More probable is a mechanism in which anion vacancies migrate to sites neighbouring the trapped gas atom; the $\mathrm{Kr}$ atom then jumps between the two vacancy sites (our calculations showed that this process was unactivated), which will be followed by the diffusion away of the vacancy into which the $\mathrm{Kr}$ was originally trapped. As anion vacancies are highly mobile in $\mathrm{UO}_{2-x}$ diffusion by this mechanism should be rapid. It would be of interest to see whether such rapid release can be observed experimentally. Our proposed mechanisms and activation energies for $\mathrm{Kr}$ release are again summarised in table IV.

4.2 Bubble Diffusion Migration. - Bubble migration, which may be either Brownian or biased (by e. g. temperature gradients) proceeds by one of three mechanism - volume or surface diffusion, or vapour transport. For intragranular fission gas bubbles, the latter mechanism is generally considered to be unimportant. The former two may be distinguished by the dependence of bubble velocity, $v$, on the bubble radius, $r$ [24] : for volume diffusion $v$ is independent of $r$, while for the surface mechanism $v \propto r^{-1}$. In table $\mathrm{V}$ we summarise the results of a number of experimental studies on bubble migration. We note that behaviour characteristic of both volume and surface mechanism has been observed.

Comparison of the experimental data on $\mathrm{UO}_{2}$ for bulk and surface diffusion suggests, however, that the surface mechanism should always dominate. Cation migration is rate determining for both volume and surface diffusion; and the cation surface diffusion coefficient is considerably greater than that for bulk cation diffusion [21]. But we have seen that volume diffusion may occur in some cases. It must therefore be possible for surface diffusion to be suppressed.

A possible suppression mechanism is suggested by a proposal in the previous section. We suggested that impurities could enhance the rate of gas migration, with the impurity remaining bound to the diffusing gas 
TABLE V

Summary of experimental data on bubble motion in nuclear fuel

Reference

-

Williamson

Cornell [3]

Manley [4]

Whapham [5]

Gulden [6]

Cornell and Bannister [7] and
Comments on experiments

\section{UO} temperature gradient. Composition of fuel unknown although extensive deviation from stoichiometry likely due to beam heating. Bubble velocity $\alpha$ radius $^{-1}$

Study similar to Williamson and Cornell with similar results. Certain bubbles, observed to be associated with small precipitates, found to move more slowly

Bubble migration in large temperature gradients produced by beam heating. Rapid migration $\left(10^{3} \AA / \mathrm{s}\right)$ observed

Experiments performed on AGR * irradiated fuel in which burn-up had proceeded to $\sim 10^{20}$ fissions $/ \mathrm{cm}^{3}$. Brownian motion of bubbles of sizes from $\sim 37 \AA$ to $70 \AA$ was observed. A number of runs at different temperatures. Bubbles were observed to be associated with precipitates in one run

Brownian motion of bubbles was observed after ion implantation using $100 \mathrm{keV} \mathrm{Kr}$ ions foils injected with $100 \mathrm{keV} \mathrm{Kr}$ ions. Migration under large

Migration mechan ism indicated

Surface diffusion indicated

Surface diffusion but
some suppression
found

No mechanistic deductions

Volume diffusion indicated

Results would be compatible with vapour transport, but appear anomalous when compared with other studies

Volume diffusion indicated

ture gradients of $\sim 10^{3} \mathrm{o} / \mathrm{cm}$ but temperature of experiments was high $\left(\sim 2000^{\circ} \mathrm{C}\right)$. Bubble velocity independent of radius. Composition claimed $\mathrm{UO}_{2.000}$ to $\mathrm{UO}_{2.005}$

Meyer [8] cating that bubbles deposited impurities as they moved
Michels and Poep- In-pile experiments on $(\mathrm{U}, \mathrm{Pu}) \mathrm{O}_{2}$ fuel with temperature gradient estimated at $\sim 5400^{\circ} \mathrm{C} / \mathrm{cm}$. Bubble trails were observed indipel [9]

$\left.{ }^{*}\right)$ Advanced Gas Reactor.

atom. If gas bubbles nucleate by migration of single gas atoms by this mechanism, then the surface of the bubbles will be coated with the impurities transported by the gas. The impurities are predominantly of low valence, and their accumulation will suppress the surface cation vacancy population. Thus if surface diffusion occurs by a vacancy mechanism, it will be suppressed by the impurities deposited at the bubble surface.

This suppression mechanism will only operate in hypostoichiometric crystals ; for, as discussed in section (4.1.1), the concerted migration of impurities and $\mathrm{Xe}$ atoms will not occur in stoichiometric or hyperstoichiometric crystals. Another factor which may limit the operation of the suppression mechanism is the magnitude of the bubble displacement. For as the bubble moves, it passes into regions of low impurity concentration with deposition of the immobile impurity shell. Thus large displacements produced by e. g. large temperature gradients will result in the bubble leaving trails of impurities, with, moreover, a change during the migration from a volume to a surface transport mechanism with a consequent acceleration of the bubble velocity.

These arguments we believe may to a large extent reconcile the apparent contradiction in the experimental data summarised in table $V$. The main difficulty, we recall, is in explaining why both volume and surface diffusion occur. Our proposals account for this in terms of different stoichiometries of the fuels or possibly different bias strengths. Such arguments appear to accord with the observed conditions of the experiments. Thus surface diffusion [3] is observed in beam heated samples where hyperstoichiometry is possible and where, in addition, large temperature gradients were present. It is significant that in one study (Manley [4]), at least some of the bubbles were observed to be associated with impurities, resulting in much slower motion, in agreement with our arguments above. 
Regarding the two experiments where volume diffusion has been observed (Gulden [6] ; Buescher and Meyer [8]) : in the former case we will have an effective hypostoichiometric fuel due to the accumulation of fission products. In the latter case, the authors report the composition of the material as being close to stoichiometry. In the former experiment the motion is Brownian while in the latter the bias is not large. The observation of volume diffusion therefore accords with our arguments. Moreover, we find good agreement between the bubble diffusion coefficients observed by Gulden [6] and that predicted using the cation diffusion coefficient given for hypostoichiometric $\mathrm{UO}_{2}$ by Matthews [21]. The agreement is less satisfactory when the results of Buescher and Meyer [8] are compared with predictions based on Matthews' value for the diffusion coefficient in the stoichiometric material. This could reflect an inadequate allowance in Matthews' expression for the variation of the cation diffusion coefficient with stoichiometry. Calculations of defect energies [13,14] suggest that Matthews' analysis may indeed underestimate the dependence of the diffusion coefficient on stoichiometry.

Finally, we should note that the experiments of Michels and Poeppel [9] detected trails behind bubbles migrating under large biases. This provides strong support for our arguments in section 4. Microprobe analyses of the chemical composition of these impurity trails would clearly be of interest.
6. Conclusions. - This paper has proposed new mechanisms for the diffusion of isolated fission gas atoms, which have been related to problems in the interpretation of data on gas bubble migration. In both areas our results have enabled us to explain a considerable range of experimental data. But problems clearly remain. The reasons for the difference between the results on gas diffusion of Felix and Miekeley [1] and Matzke [2] must be discovered. We have suggested the presence in the latter studies of traps produced by radiation damage, as a possible explanation. For the present, however, this proposal must be tentative.

An obvious suggestion for further experimental work concerns $\mathrm{Kr}$ diffusion. We have suggested that release rates for $\mathrm{Kr}$ in hypostoichiometric $\mathrm{UO}_{2}$ might differ very considerably from those observed for Xe. An experimental study to test this prediction would clearly be of great interest. In the field of bubble migration further work is also clearly needed. In particular experiments specifically designed to test separately the effects of stoichiometry, impurity concentrations and bias strength - factors which our discussion has stressed as being of particular importance in determining the mechanisms of bubble migration - would be of great value in advancing our understanding of bubble migration mechanisms in nuclear fuels.

Acknowledgment. - We are grateful to Dr. A. B. Lidiard for several helpful discussions.

References

[1] FÉlix, F. W. and Miekeley, W., J. Nucl. Mater, 42 (1972) 297.

[2] Matzke, Hj., Physics of Ionised Gases (ed. Navinsek B.), Proc. Summer School Hercegnovi, Yugoslavia, p. 396.

[3] Williamson, G. K. and Cornell, R. M., J. Nucl. Mater. 13 (1964) 278.

[4] Manley, A. J., J. Nucl. Mater. 26 (1968) 244.

[5] Whapham, A. D., Nucl. Appl. 2 (1966) 123.

[6] Gulden, M. E., J. Nucl. Mater. 23 (1967) 30.

[7] Cornell, R. M. and Bannister, G. H., Proc. Brit. Ceramic Soc. 7 (1967) 355.

[8] Buescher, B. J. and Meyer, R. O., J. Nucl. Mater. 48 (1973) 143.

[9] Michels, L. C. and Poeppel, R. B., Appl. Phys. 44 (1973) 1003.

[10] Catlow, C. R. A., Corish, J., Diller, K. M., JacoBs, P. W. M. and Norgetr, M. J., J. Physique Colloq. 37 (1976) C7, this issue.

[11] Catlow, C. R. A., James, R. and Norgett, M. J., J. Physique Colloq. 37 (1976) C7, this issue.
[12] Catlow, C. R. A. and Norgett, M. J., J. Phys. C 6 (1973) 1325.

[13] Catlow, C. R. A. and Lidiard, A. B., Proc. Symp. on Thermodynamics of Reactor Materials, IAEA, Vienna (1974), Vol. II, p. 27.

[14] Catlow, C. R. A., Proc. R. Soc. London, in press.

[15] Norget,, M. J. and Lidiard, A. B., Phil. Mag. 18 (1968) 1193.

[16] Wedepohl, P. T., Proc. Phys. Soc. 92 (1967) 79.

[17] Gordon, R. G. and KIM, Y. S., J. Chem. Phys. 56 (1972) 3122.

[18] Catlow, C. R. A., Diller, K. M. and Norgett, M. J., J. Phys. C., in press.

[19] CATLOW, C. R. A., UKAEA Report AERE, TP669.

[20] Dick, B. G. and Overhauser, A. W., Phys. Rev. 112 (1958) 90.

[21] MATthews, J. R., UKAEA report AERE M2643.

[22] Norgett, M. J., J. Phys. C 4 (1971) 1284.

[23] Catlow, C. R. A., Lidtard, A. B. and Norgett, M. J., J. Phys. C 8 (1975) L-435.

[24] Nichols, F. A., J. Nucl. Mater. 30 (1969) 143. 


\section{DISCUSSION}

Hj. MATZKE. - I would like to congratulate you on the elegant calculation techniques and on your interesting results which certainly are valuable to better understand different mechanisms contributing to gas release from $\mathrm{UO}_{2}$. However, I would like to point out that an acceptable model should allow for all reliable observations. Certainly, a more comprehensive comparison would be very helpful in deciding under which conditions your suggested mechanisms are applicable.

Also, one should be aware of the fact that one is not really comparing with conventional diffusion coefficients but rather with values deduced from release curves. The time dependence is known, but not the depth dependence. One should therefore very carefully check whether release values really represent unperturbed volume diffusion. Just one example : if release from $\mathrm{UO}_{2-x}$ is slower than release from $\mathrm{UO}_{2}$ it could also be due to the known tendency of oxygen vacancies in $\mathrm{UO}_{2-x}$ to cluster in planar defects in an attempt to form a shear structure. The gas could get trapped in the strain field of the surrounding dislocations. Unless we know more about the thermal stability of clusters and kinetics of detrapping, we cannot a priori discard such mechanism. Similarly, in $\mathrm{UO}_{2+x}$, one is dealing with three, two or single phased fields with cluster and long range order, depending on $x$ and temperature. It would be surprising if a single mechanism would explain all of the observed behaviour.

C. R. A.CATLOW. - I accept your remarks concerning the analysis of the experimental data. In presenting any models, however, I consider that it is necessary to attempt to tie them in with available experimental results. But I fully agree that a more complete analysis of the data is certainly now required. 